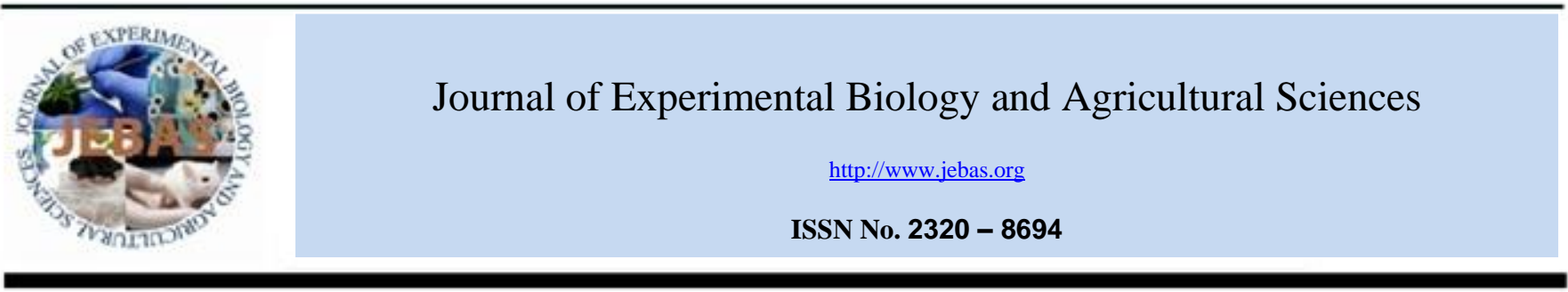

\title{
GROWTH AND DEVELOPMENT INHIBITORY ACTIVITIES OF MEDICINAL PLANT OILS AGAINST LEMON BUTTERFLY
}

\section{Deepika Chauhan ${ }^{1, *}$ and Poonam Srivastava ${ }^{2}$}

\author{
${ }^{1}$ Assistant Professor, College of Horticulture, VCSG-UUHF Bharsar \\ ${ }^{2}$ Assistant Professor, GBPUA\&T, Pantnagar, Department of Entomology, G.B. Pant University of Agriculture and Technology, Pantnagar
}

Received - January 25, 2017; Revision - March 27, 2017; Accepted - April 14, 2017

Available Online - May 12, 2017

DOI: http://dx.doi.org/10.18006/2017.5(2).258.263

\author{
KEYWORDS \\ Growth and development \\ Medicinal oils \\ Papilio demoleus
}

\begin{abstract}
Present study was carried out to access the effect of $2 \%$ concentration of some medicinal plant oils on growth and development inhibitory activities of lemon butterfly, Papilio demoleus. The observations on mean weight gain/larva at two days after feeding (DAF) specified that minimum weight gain $(0.018 \mathrm{~g} / \mathrm{larva})$ or highest reduction in weight gain over control was reported in diet supplemented with Cymbopogan flexuosus- Chirharit (59.10\%) and it was followed by Jatropha curcus $(56.82 \%)$. All the plant oils except Geranium were found effective in reducing the larval weight significantly over control $(0.0260 \mathrm{~g})$ at 4 days after feeding. The plant oils used in the present investigation revealed different level of toxicity against $P$. demoleus. Like weight gain, highest larval mortality (\%) was reported from the $C$. flexuosus- Chirharit (53.33\%). Further, a significant reduction in the pupal weight was also reported and among various tested oils. Maximum reduction (59.17\%) in pupal weight was detected in C. flexuosusChirharit while minimum reduction $(23.34 \%)$ was observed in Geranium essential oils. In the present investigation, C. flexuosus- Chirharit, C. flexuosus- Krishna, Mentha arvensis and J. curcus was effective in reducing growth and developmental parameters of 3 days old P. demoleus larvae.
\end{abstract}

* Corresponding author

E-mail: deepika40865@gmail.com (Deepika Chauhan)

Peer review under responsibility of Journal of Experimental Biology and Agricultural Sciences.

Production and Hosting by Horizon Publisher India [HPI] (http://www.horizonpublisherindia.in/).

All rights reserved.
All the article published by Journal of Experimental Biology and Agricultural Sciences is licensed under a Creative Commons Attribution-NonCommercial 4.0 International License Based on a work at www.jebas.org.

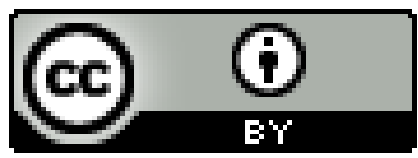




\section{Introduction}

The lemon butterfly, $P$. demoleus is the most notorious and caustic pest of citrus plantation all over the world. It caused severe damage to citrus family under field conditions by consuming enormous amount of plants throughout later stages of their growth (Yunus \& Munir, 1972). Among the various insect pests that attack citrus, the citrus butterfly, $P$. demoleus Linnaeus is a regular pest in nurseries, young seedlings and on new flush of full grown up tress and it is considered as the most important foliage feeding insect in India (Bhutani \& Jotwani, 1975; Srivastava, 1993; Vattikonda \& Sangam, 2016). It defoliates citrus plants and are particularly damaging to plants in the seed beds and nurseries. The first choice of larvae is young plants of 1-2 feet high and is capable of completely defoliating nursery groves (Singh, 1993; Matsumoto, 1996; Narayanamma et al., 2001). Distribution capability and ability for rapid population rise make $P$. demoleus a potentially severe. Subsequently, appropriate management of $P$. demoleus could play an important economic impact on production and prosperity of the citrus crop. For proper management of any insect pest, it is vital to be familiar with the biology, ecology and feeding performance of the insect. Moreover the utilization of synthetic insecticides has caused several environmental and health concerns.

Therefore, there is a need of new natural strategies headed for insect pest management which consists of botanicals for example plant products and plant essential oils to combat residue and resistance problem. In recent years, great importance has been provided on the use of natural products, which are effective, protected, non-hazardous, inexpensive and eco-friendly substitute to the predictable control of insects by artificial pesticides. Previous studies have specified that antifeedant compounds obtained from roots, seeds, leaves, flowers and fruits of the plants could be employed for effective biocompounds and exert toxicity against the metamorphosis and development of the injurious insects. The botanical pesticides are generally pest-specific and are relatively less harmless to non-target organisms including man and natural enemies of insect pests. These are also environmentally eco-friendly, degrade rapidly in sunlight, air, and moisture, so they are less persistence in the environment, and are rapid in action to the insect pests (Vattikonda \& Sangam, 2016). There is an urgent need for the expansion of safer, alternative crop protectants such as botanical insecticides and antifeedants. Pandey et al. (2012) evaluated the efficacy of leaf extracts of Eucalyptus globules, Ageratum conyzoids and Allium sativum against fifth instar larvae of $P$. demoleus.

Plants are basis of natural essences that can be exploited in the advancement of environmentally safe methods for insect control (Sadek, 2003). However, there is inadequate literature is available regarding the effect of plant essential oils on $P$. demoleus. Therefore, in the present exploration some medicinal plants were determined for their growth and development inhibitory activities against lemon butterfly, $P$. demoleus in ecofriendly manner.

\section{Materials and Methods}

\subsection{Experimental setup and observations}

This experiment was conducted in the department of Entomology, G. B. Pant University of Agriculture and Technology, Pantnagar, Uttarakhand (India). The used essential oil samples were collected from Medicinal Research and Developmental Centre (MRDC), Pantnagar. In the present study ten plant oils viz., Lemon grass- Chirharit, (Cymbopogan flexuosus Stapf); Lemon grass- Krishana, (Cymbopogan flexuosus Stapf); Jatropha, (Jatropha curcas L.); Citronella grass, Ganjri, (Cymbopogan nardus L.); Eucalyptus, Nilgiri, (Eucalyptus citriodora Hook); Lippia, (Lippia alba L.); Lemon tulsi, (Ocimum tenuiflorum); Levender, (Lavandula angustifolia L.); Fennel, (Foeniculum vulgare Mill.); Mint, (Mentha arvensis L.); Palmrosa, (Cymbopogan martinii (Roxb.) Wats.); Cranesbills, (Geranium sp. L.) were tested at $2 \%$ concentration for their consequences on growth and developmental constraints of larvae of $P$. demoleus. The larvae were used for conducting the various experiments (Chauhan, 2016).

The experiment was executed under laboratory conditions in the large petri plates supplied with two layers of humid filter paper to make sure suitable relative humidity within the petri plates. The laboratory raised larvae were employed for carrying out experimentations on growth and development. The desired concentrations of used essential oil were got ready in acetone while control contains only acetone. Each of the treatments was replicated three times and for each replication 10 larvae of same age group and size were taken in each petridish. For imposing treatment, the equal size lemon leaves were immersed into the particular plant oil concentration for 2-3 minutes, air dried and then put into the petri plates. The larvae were given food with fresh treated leaves continuously for three days and thereafter with fresh untreated leaves until pupation.

The observations were recorded on following parameterslarval weight (g), larval period (d), pupal period (d), and adult emergence $(\%)$. Here various growth parameters were measured as mentioned below:

1. Weight gain/larva $=$ Wt. of single larva $2 / 3 \mathrm{DAF}-$ Initial weight of single larva.

2. Reduction in weight gain over control $=100-$ $\left(\frac{\mathrm{T}}{\mathrm{C}} \times 100\right)$ (Vaitheeswaran, 2009)

Where $\mathrm{T}=$ weight gain in treatment; $\mathrm{C}=$ Weight gain in control

3. Mortality $(\%)=\underline{\text { Number of dead larvae }} \times 100$ Total larvae taken

Journal of Experimental Biology and Agricultural Sciences

http://www.jebas.org 
While various development parameters such as larval period, pupal period and adult emergence were measured as mentioned below:

1. Larval period (d): Number of days required from hatching to full grown larva

2. Pupal period (d): The period between the commencement of pupation and adult emergence.

3. Adult emergence (\%)

$=\quad$ Number of healthy adults emerged $\times 100$ Number of healthy pupae formed

\subsection{Experimental Design and Statistical analysis}

All the laboratory tests were conducted in completely randomized design (CRD) (Gomez \& Gomez, 1984) and the data was evaluated by One Way Analysis of Variance (ANOVA) following Snedecor \& Cochran (1967). The means were separated by means of Duncan Multiple Range Test (DMRT) (Duncan, 1955) based SPSS16 (Statistical Product and Service Solutions) computer programme

\section{Results}

3.1 Growth and development parameters

Results of mean weight gain/larva at two days after feeding (DAF) indicated that minimum weight gain $(0.018 \mathrm{~g} / \mathrm{larva})$ or maximum reduction in weight gain of $P$. demoleus over control was exhibited by $C$. flexuosus $(59.10 \%)$ and it was followed by J. curcus (56.82\%), C. flexuosus- Krishna (54.55\%), L. angustifolia (52.28\%), M. arvensis (50.0\%), C. nardus (43.19\%), O. tenuiflorum (40.91\%), L. alba (34.10\%), E. citriodora (27.54\%), F. vulgare $(20.46 \%), \quad$ Geranium (11.37\%) and C. martinii (9.10\%)(table 1).

Four days after feeding (4 DAF) also all the plant oils except Geranium were effective in reducing the larval weight significantly over control $(0.0260 \mathrm{~g})$. Here also maximum reduction in weight gain or minimum weight gain $(0.100 \mathrm{~g} /$ larva) was reported in C. flexuosus $(61.54 \%)$ essential oil. Rest of the essential oil treatments are also showing similar trends.

Table 1 Effect of different medicinal plant oils on growth of 3 day old larvae of $P$. demoleus.

\begin{tabular}{|c|c|c|c|c|c|c|}
\hline $\begin{array}{l}\text { SI. } \\
\text { No. }\end{array}$ & $\begin{array}{c}\text { Plant species } \\
\text { Scientific name (Common } \\
\text { name) }\end{array}$ & $\begin{array}{l}\text { Mean wt } \\
\text { gain/larva } \\
2 \mathrm{DAF}(\mathrm{g})\end{array}$ & $\begin{array}{c}\text { Reduction in wt } \\
\text { gain/larva over } \\
\text { control } 2 \text { DAF } \\
(\%)\end{array}$ & $\begin{array}{c}\text { Mean wt } \\
\text { gain/larva 3+1 } \\
\text { DAF }\end{array}$ & $\begin{array}{c}\text { Reduction in wt } \\
\text { gain/larva over } \\
\text { control 3+1 DAF } \\
(\%)\end{array}$ & $\begin{array}{c}\text { Larval } \\
\text { mortality }\end{array}$ \\
\hline 1. & $\begin{array}{l}\text { C. flexuosus Stapf. (Lemon } \\
\text { grass-Chirharit) }\end{array}$ & $0.018 \pm 0.001^{\mathrm{a}}$ & $59.10 \pm 1.21^{\mathrm{i}}$ & $0.100 \pm 0.12^{\mathrm{a}}$ & $61.54 \pm 1.1^{\mathrm{h}}$ & $53.33 \pm 1.73^{\mathrm{g}}$ \\
\hline 2. & $\begin{array}{l}\text { C. flexuosus Stapf.( Lemon } \\
\text { grass-Krishana) }\end{array}$ & $0.020 \pm 0.005^{\mathrm{a}}$ & $54.55 \pm 2.28^{\mathrm{gh}}$ & $0.150 \pm 0.08^{\mathrm{a}}$ & $57.69 \pm 0.01^{\mathrm{gh}}$ & $30.0 \pm 2.8^{\mathrm{de}}$ \\
\hline 3. & Jatropha curcas L. (Jatropha) & $0.019 \pm 0.005^{\mathrm{a}}$ & $56.82 \pm 0.68^{\mathrm{hi}}$ & $0.150 \pm 0.08^{\mathrm{a}}$ & $42.31 \pm 0.005^{\mathrm{e}}$ & $33.33 \pm 1.7^{\mathrm{ef}}$ \\
\hline 4. & $\begin{array}{l}\text { Cymbopogan nardusL. } \\
\text { (Citronella grass, Ganjri) }\end{array}$ & $0.0250 \pm .002^{\mathrm{abc}}$ & $43.19 \pm 1.78^{\mathrm{e}}$ & $0.175 \pm 0.014^{\mathrm{a}}$ & $32.7 \pm 0.02^{\mathrm{cd}}$ & $26.66 \pm 3.46^{\text {cd }}$ \\
\hline 5. & $\begin{array}{l}\text { Eucalypts citriodoraHook. } \\
\text { (Eucalyptus,Nilgiri) }\end{array}$ & $0.030 \pm 0.005^{\mathrm{a}}$ & $27.54 \pm 0.55^{\mathrm{c}}$ & $0.202 \pm 0.001^{\mathrm{a}}$ & $22.31 \pm 1.16^{\mathrm{b}}$ & $26.66 \pm 1.41^{\text {cd }}$ \\
\hline 6. & Lippia alba L.(Lippia) & $0.029 \pm 0.005^{\mathrm{abcd}}$ & $34.10 \pm 2.31^{\mathrm{d}}$ & $0.165 \pm 0.002^{\mathrm{a}}$ & $36.54 \pm 0.02^{\mathrm{d}}$ & $16.66 \pm 0.57^{\mathrm{b}}$ \\
\hline 7. & $\begin{array}{l}\text { Ocimum tenuiflorum } \\
\text { (Lemon tulsi, Tulsi,) }\end{array}$ & $0.0260 .003^{\mathrm{abcd}}$ & $40.91 \pm 0.58^{\mathrm{e}}$ & $0.117 \pm 0.004^{\mathrm{a}}$ & $55.0 \pm 4.41^{\mathrm{g}}$ & $23.33 \pm 1.73^{\mathrm{c}}$ \\
\hline 8. & $\begin{array}{l}\text { Lavandula angustifolia } \\
\text { Levender, }\end{array}$ & $0.021 \pm 0.005^{\mathrm{ab}}$ & $52.28 \pm 0.03^{\mathrm{fg}}$ & $0.126 \pm 0.001^{\mathrm{a}}$ & $51.54 \pm 0.60^{f}$ & $16.66 \pm 0.76^{\mathrm{b}}$ \\
\hline 9. & $\begin{array}{l}\begin{array}{l}\text { Foeniculum } \\
\text { (Fennel,) }\end{array} \\
\text { vulgare }\end{array}$ & $0.035 \pm 0.002^{\mathrm{abcd}}$ & $20.46 \pm 0.612^{\mathrm{b}}$ & $0.185 \pm 0.002^{\mathrm{a}}$ & $28.85 \pm 0.34^{\mathrm{c}}$ & $6.6 \pm 0.34^{\mathrm{a}}$ \\
\hline 10. & Mentha arvensis L.( Mint) & $0.022 \pm 0.001^{\mathrm{abc}}$ & $50.0 \pm 2.8^{f}$ & $0.132 \pm 0.001^{\mathrm{a}}$ & $49.24 \pm 0.023^{\mathrm{f}}$ & $36.66 \pm 3.43^{\mathrm{f}}$ \\
\hline 11. & $\begin{array}{l}\text { C. martinii (Roxb.) Wats. } \\
\text { (Palmrosa, }\end{array}$ & $0.040 \pm 0.012^{\mathrm{cd}}$ & $9.10 \pm 0.05^{\mathrm{a}}$ & $0.201 \pm 0.005^{\mathrm{a}}$ & $22.70 \pm 0.65^{\mathrm{b}}$ & $6.66 \pm 0.34^{\mathrm{a}}$ \\
\hline \multirow[t]{5}{*}{12.} & Geranium sp. L.(Cranesbills) & $0.039 \pm 0.002^{\mathrm{bcd}}$ & $11.37 \pm 0.04^{\mathrm{a}}$ & $0.230 \pm 0.017^{\mathrm{a}}$ & $11.54 \pm 0.34^{\mathrm{a}}$ & $6.66 \pm 0.34^{\mathrm{a}}$ \\
\hline & Control & $0.044 \pm 0.002^{\mathrm{d}}$ & - & $0.260 \pm 0.002^{\mathrm{a}}$ & - & - \\
\hline & \multirow{3}{*}{$\begin{array}{l}\mathrm{SEM} \pm \\
\mathrm{CD} \text { at } 5 \% \\
\text { F-value }\end{array}$} & 0.005 & 1.44 & 2.93 & 1.40 & 1.92 \\
\hline & & 0.0163 & 4.21 & 8.53 & 4.08 & 5.62 \\
\hline & & $*$ & $* *$ & ns & $* *$ & $* *$ \\
\hline
\end{tabular}

Means $( \pm$ SE) followed by same letters do not differ significantly by DMRT $(\mathrm{p}=0.05 \%)$; DAF- Days after feeding, $3+1 \mathrm{DAF}=\mathrm{Feeding}$ with treated leaves $(3 \mathrm{~d})+$ untreated leaves $(1 \mathrm{~d}) ; *=$ Significant, $* *=$ highly significant, $\mathrm{ns}=$ non-significant 
Table 2 Effect of different medicinal plant oils on development of 3 day old larvae of $P$. demoleus

\begin{tabular}{|c|c|c|c|c|c|c|c|c|c|}
\hline $\begin{array}{l}\dot{0} \\
\dot{\mathrm{z}}\end{array}$ & 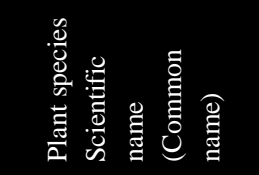 & 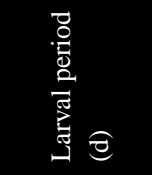 & 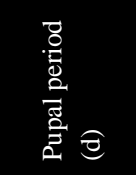 & 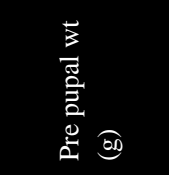 & 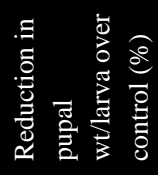 & $\sum_{B} \widehat{Q}$ &  & 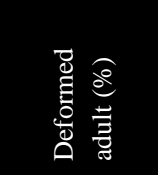 & 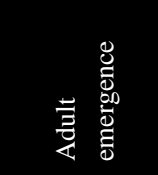 \\
\hline 1. & $\begin{array}{l}\text { C. flexuosus Stapf. } \\
\text { (Lemon grass- } \\
\text { Chirharit) }\end{array}$ & $13.9 \pm 0.54^{\mathrm{a}}$ & $8.10 \pm 0.05^{\mathrm{a}}$ & $0.490 \pm 0.005^{\mathrm{a}}$ & $59.17 \pm 0.005^{\mathrm{g}}$ & $16.66 \pm 0.005^{\mathrm{e}}$ & $30.00 \pm 0.57^{\mathrm{a}}$ & $10.0 \pm 0.93^{\mathrm{d}}$ & $26.66 \pm 1.73^{\mathrm{a}}$ \\
\hline 2. & $\begin{array}{l}\text { C. flexuosus Stapf.( } \\
\text { Lemon grass- } \\
\text { Krishana) }\end{array}$ & $14.9 \pm 0.29^{\mathrm{a}}$ & $8.5 \pm 0.28^{\mathrm{ab}}$ & $0.700 \pm 0.05^{\mathrm{bc}}$ & $41.67 \pm 0.005^{\mathrm{d}}$ & $16.66 \pm 1.15^{\mathrm{e}}$ & $46.66 \pm 1.52^{\mathrm{b}}$ & $13.33 \pm 0.52^{\mathrm{e}}$ & $43.33 \pm 1.72^{\mathrm{bc}}$ \\
\hline 3. & $\begin{array}{l}\text { Jatropha curcas L. } \\
\text { (Jatropha) }\end{array}$ & $14.5 \pm 0.69^{\mathrm{a}}$ & $8.5 \pm 0.28^{\mathrm{ab}}$ & $0.780 \pm 0.005^{\mathrm{cd}}$ & $35.0 \pm 0.57^{\mathrm{c}}$ & $13.33 \pm 0.005^{\mathrm{d}}$ & $50.0 \pm 0.60^{c}$ & $13.33 \pm 0.08^{\mathrm{e}}$ & $46.66 \pm 1.73^{\mathrm{c}}$ \\
\hline 4. & $\begin{array}{l}\text { Cymbopogan } \\
\text { nardusL.(Citronella } \\
\text { grass, Ganjri) }\end{array}$ & $14.15 \pm 0.002^{\mathrm{a}}$ & $9.0 \pm 0.60^{\mathrm{ab}}$ & $0.810 \pm 0.005^{\text {cd }}$ & $32.5 \pm 1.18^{\mathrm{c}}$ & $10.0 \pm 0.66^{\mathrm{c}}$ & $66.67 \pm 1.52^{\mathrm{e}}$ & $3.33 \pm 0.57^{\mathrm{b}}$ & $66.67 \pm 0.57^{\mathrm{e}}$ \\
\hline 5. & $\begin{array}{l}\text { Eucalyptus } \\
\text { citriodoraHook. } \\
\text { (Eucalyptus,Nilgiri) }\end{array}$ & $14.30 \pm 0.54^{\mathrm{a}}$ & $8.9 \pm 0.11^{\mathrm{ab}}$ & $0.800 \pm 0.005^{\mathrm{cd}}$ & $33.34 \pm 1.73^{\mathrm{c}}$ & $6.66 \pm 0.0 .58^{\mathrm{b}}$ & $70.0 \pm 0.94^{\mathrm{f}}$ & $3.33 \pm 1.15^{\mathrm{b}}$ & $70.00 \pm 0.94^{\mathrm{e}}$ \\
\hline 6. & $\begin{array}{l}\text { Lippia alba } \\
\text { L.(Lippia) }\end{array}$ & $14.15 \pm 0.68^{\mathrm{a}}$ & $8.9 \pm 0.57^{\mathrm{ab}}$ & $0.692 \pm 0.054^{\mathrm{bc}}$ & $42.34 \pm 1.00^{\mathrm{de}}$ & $13.33 \pm 1.72^{\mathrm{e}}$ & $70.0 \pm 0.94^{\mathrm{f}}$ & $3.33 \pm 0.05^{\mathrm{b}}$ & $70.0 \pm 0.94^{\mathrm{d}}$ \\
\hline 7. & $\begin{array}{l}\text { Ocimum } \\
\text { tenuiflorum L. } \\
\text { (Lemon tulsi, } \\
\text { Tulsi,) }\end{array}$ & $13.26 \pm 1.15^{\mathrm{a}}$ & $8.1 \pm 0.52^{\mathrm{a}}$ & $0.495 \pm 0.05^{\mathrm{a}}$ & $58.75 \pm 0.54^{g}$ & $13.33 \pm 0.58^{d}$ & $63.34 \pm 0.57^{\mathrm{c}}$ & $6.66 \pm 0.58^{\mathrm{c}}$ & $56.66 \pm 1.15^{\mathrm{d}}$ \\
\hline 8. & $\begin{array}{l}\text { Lavandula } \\
\text { angustifolia L.( } \\
\text { Levender,) }\end{array}$ & $13.5 \pm 0.60^{\mathrm{a}}$ & $8.2 \pm 0.50^{\mathrm{a}}$ & $0.562 \pm 0.018^{\mathrm{ab}}$ & $53.17 \pm 0.33^{\mathrm{f}}$ & $6.66 \pm 0.53^{\mathrm{b}}$ & $70.0 \pm 0.59^{\mathrm{f}}$ & $3.33 \pm 0.50^{\mathrm{b}}$ & $66.66 \pm 2.33^{\mathrm{e}}$ \\
\hline 9. & $\begin{array}{l}\text { Foeniculum } \\
\text { vulgare Mill. } \\
\text { (Fennel,) }\end{array}$ & $14.25 \pm 1.0^{\mathrm{a}}$ & $9.6 \pm 0.54^{\mathrm{ab}}$ & $0.780 \pm 0.011^{\mathrm{cd}}$ & $35.0 \pm 0.44^{\mathrm{c}}$ & $3.33 \pm 0.50^{\mathrm{a}}$ & $90.0 \pm 0.94^{\mathrm{g}}$ & $0.00 \pm 0.00^{\mathrm{a}}$ & $40.0 \pm 0.54^{\mathrm{b}}$ \\
\hline 10. & $\begin{array}{l}\text { Mentha arvensis } \\
\text { L.( Mint) }\end{array}$ & $13.9 \pm 1.04^{\mathrm{a}}$ & $8.9 \pm 0.33^{\mathrm{ab}}$ & $0.650 \pm 0.026^{\mathrm{ab}}$ & $45.84 \pm 2.88^{\mathrm{e}}$ & $16.66 \pm 1.92^{\mathrm{e}}$ & $46.67 \pm 0.99^{b}$ & $16 \pm .66 \pm 0.57^{\mathrm{f}}$ & $46.67 \pm 0.52^{\mathrm{c}}$ \\
\hline 11. & $\begin{array}{l}\text { C. martinii (Roxb.) } \\
\text { Wats. (Palmrosa,) }\end{array}$ & $14.10 \pm 0.60^{\mathrm{a}}$ & $9.26 \pm 0.57^{\mathrm{ab}}$ & $0.800 \pm 0.05^{\mathrm{cd}}$ & $33.34 \pm 1.73^{\mathrm{c}}$ & $3.33 \pm 1.73^{\mathrm{a}}$ & $90.0 \pm 0.52^{\mathrm{g}}$ & $6.66 \pm 0.57^{\mathrm{c}}$ & $86.66 \pm 0.058^{\mathrm{f}}$ \\
\hline \multirow[t]{5}{*}{12.} & $\begin{array}{l}\text { Geranium sp. } \\
\text { L.(Cranesbills) }\end{array}$ & $14.28 \pm 0.57^{\mathrm{a}}$ & $9.62 \pm 0.32^{\mathrm{ab}}$ & $0.920 \pm 0.052^{\mathrm{d}}$ & $23.34 \pm 1.72^{\mathrm{b}}$ & $3.33 \pm 1.72^{\mathrm{a}}$ & $90.0 \pm 0.94^{\mathrm{h}}$ & $0.00 \pm 0.00^{\mathrm{a}}$ & $90.00 \pm 0.05^{\mathrm{f}}$ \\
\hline & Control & $14.26 \pm 0.50^{\mathrm{a}}$ & $9.0 \pm 0.8^{\mathrm{b}}$ & $1.20 \pm 0.15^{\mathrm{e}}$ & $0.00 \pm 0.00^{\mathrm{a}}$ & $3.33 \pm 0.00^{\mathrm{a}}$ & $96.67 \pm 1.72^{\mathrm{h}}$ & $0.00 \pm 0.00^{\mathrm{a}}$ & $96.67 \pm 0.052^{\mathrm{g}}$ \\
\hline & \multirow{3}{*}{$\begin{array}{l}\mathrm{SEM} \pm \\
\mathrm{CD} \text { at } 5 \% \\
\text { F-value }\end{array}$} & 0.051 & 0.844 & 0.028 & 0.226 & 0.160 & 0.452 & 0.160 & 0.320 \\
\hline & & 0.149 & 2.45 & 0.082 & 0.659 & 0.465 & 1.31 & 0.465 & 0.931 \\
\hline & & $* *$ & ns & ** & $* *$ & $* *$ & $* *$ & $* *$ & $* *$ \\
\hline
\end{tabular}

Means $( \pm$ SE) followed by same letters do not differ significantly by DMRT $(\mathrm{p}=0.05 \%)$; TLM- Terminal larval mortality, $* *=$ Highly significant, ns- non-significant

Further, minimum reduction in weight gain $(11.54 \%)$ or maximum weight gain $(0.230 \mathrm{~g} / \mathrm{larva})$ over the control $(0.0260 \mathrm{~g} / \mathrm{larva})$ was observed in Geranium essential oil (table 1). The plant oils taken in the present investigation reflected different level of toxicity against $P$. demoleus. Like weight gain, maximum larval mortality (\%) was reported in the $C$. flexuosus- Chirharit $(53.33 \%)$ and it was followed by $M$. arvensis $(36.66 \%)$, J. curcus $(33.3 \%)$, C. flexuosus-Krishna $(30.0 \%)$. Further, in case of mortality, $26.66 \%$ mortality was observed in $C$. nardus and E. citriodora which was followed by $O$. tenuiflorum (23.33) and L. alba (16.66\%). Minimum mortality (6.66\%) was observed in F. vulgare, C. martinii and Geranium while in control no mortality was observed (table 1).

\subsection{Effect of essential oils on the development of P. demoleus}

Plant essential oils such as C. flexuosus (13.9d), L. angustifolia (13.5d), M. arvensis (13.9d), C. martinii (14.10d), C. nardus (14.15d) and $F$. vulgare $(14.25 \mathrm{~d})$ at $2 \%$ concentration decreased the larval period however C. flexuosus (14.9d), $J$. curcus (14.5d), E. citriodora (14.3d) and Geranium (14.28d) increased the larval period of $P$. demoleus as compared to 
control (14.26d). Lowest larval period was observed in $O$. tenuiflorum (13.26d), however the differences among all the treatments were non-significant (table 2).

The terminal larval mortality (\%) was reported maximum (16.66\%) in the C. flexuosus, C. flexuosus and M. arvensis and it was pursued by $J$. curcus $=L$. alba $=O$. tenuiflorum (13.33\%), C. nardus (10.0\%), E. citriodora $=$ L. angustifolia $(6.66 \%)$ while minimum larval mortality was observed in $F$. vulgare (3.33\%). Maximum pupal period was observed in Geranium (9.62d) and it was followed by $F$. vulgare (9.6d), $C$. martini (9.26d) as compared of control (9.0d) while minimum pupal period was observed in $O$. tenuiflorum and $C$. flexuosusChirharit (8.10d). Other oils L. angustifolia (8.2d), $C$. flexuosus-Krishna (8.5d), J. curcus (8.5d), E. citriodora (8.9d) , L. alba (8.9d) and M. arvensis (8.9d) also showed the minimum range of pupal period while $C$. nardus recorded $9.0 \mathrm{~d}$ pupal period similar to control (table 2).

All the plant oils caused a significant reduction in pupal weight over control. Minimum pupal wt $(0.490 \mathrm{~g})$ or maximum reduction $(59.17 \%)$ was observed in C. flexuosus while minimum reduction $(23.34 \%)$ was observed in Geranium. The percent pupation was significantly reduced by the application of all plant oils. Higher percent pupation was observed in control $(96.67 \%)$ which was followed by Geranium $=$ C. martini and F.vulgare (90.0\%). Among various tested treatments, minimum pupation was recorded in C. flexuosus (30.0\%). All the plant oils except $F$. vulgare and Geranium caused the development of deformed adult. Maximum per cent of deformed adult was recorded by $C$. flexuosus and J. curcus $(13.33 \%)$. The percent adult emergence was adversely affected by some plant oils viz., C. flexuosus (26.66\%), F. vulgare (40.0\%), C. flexuosus (43.33\%), M. arvensis (46.67\%), J.curcus (46.66\%), O. tenuiflorum (56.66\%), C. nardus (66.67\%), L. angustifolia (66.66\%), E. citrodora (70.0\%), L. alba $(70.0 \%)$, C. martinii (86.66\%) and Geranium (90.0\%). Highest adult emergence was observed in control (96.67\%).

\section{Discussions}

Results of study suggested that essential oils have signification effect in the growth and development of the $P$. demoleus. In present investigation, essential oil of $C$. flexuosus, $M$. arvensis and $J$. curcus was effective in reducing feeding and developmental parameters in $3 \mathrm{~d}$ old larvae of $P$. demoleus. Similar results were observed by Meshram et al. (1996) when they tried that petroleum ether extracts of two medicinal plant viz., A. indica and Jatropha curcas at different concentrations against the third instar larvae of $P$. demoleus under laboratory conditions. Result of this study revealed that 0.3 per cent petroleum ether extract of $A$. indica deters the larvae from feeding. The average leaf area consumed in treated leaves by the larvae was found to be 33.77 per cent as compared to 85.85 per cent in control. Further, Pandey et al. (2012) evaluated the efficacy of leaf extracts of Eucalyptus globules, Ageratum conyzoids and Allium sativum against fifth instar larvae of $P$. demoleus and reported abnormalities in morphogenetic development. Plant extracts also caused significant reduction in larval body weight and relatively higher reduction in body weight was reported in E. globules treated larvae. Later on, Vattikonda \& Sangam (2016) established antifeedant activity of azadirachtin (a secondary metabolite of Azadirachta indica) against fourth instar larvae of $P$. demoleus.

Similarly, insecticidal properties of $L$. alba (Tripathi et al., 2002), Jatropha curcas (Murthy et al., 2007), Ocimum basilicum, Cymbopogon winterianus, Vitex negundo, Callistemon lanceolatus, Eucalyptus sp., Lantana camara, Nerium oleander, O. sanctum (Sharma et al., 2001) essential oil was reported against Spodoptera litura third instar larvae. Likewise, Pandey (2011) performed an experiment to find out antifeedant activity of seven plant oils (1\% and $2 \%$ concentration in acetone) viz., C. flexuosus, Ocimum tenuiflorum, Cymbopogan winterianus Bio 13; C. flexuosus; Vetiveria zizanioides, Pogostemon patchouli and Eucalyptus citridora against $7 \mathrm{~d}$ old larvae of $S$. litura and revealed that all plant oils except, $P$. patchouli and E. citriodora showed promising reduction in larval feeding. The outcome of the present study would be helpful in endorsing the exploration of such type of studies with the aim for enlargement of new means for pest control based on natural products.

\section{Acknowledgement}

Authors are grateful to the Medicinal Plant Research and Development Centre (MRDC) of G.B. Pant University of Agriculture and Technology, Pantnagar for making available the plant oils.

\section{Conflict of interest}

Authors would hereby like to declare that there is no conflict of interests that could possibly arise.

\section{References}

Bhutani BK, Jotwani MG (1975) Trends in the control of insect pests of fruit crops in India. Pesticide 9: 139-149.

Chauhan D (2016) Studies on population dynamics of citrus psylla and biological attributes of lemon butterfly alongwith their management. Ph.D Thesis submitted to the G.B. Pant University of Agriculture and Technology, Pantnagar. $285 \mathrm{p}$.

Duncan DB (1955) Multiple range and multiple F- tests. Biometrics 11: 1-42.

Gomez KA, Gomez AA (1984) Statistical procedures for agricultural research. $2^{\text {nd }}$ edition. John Wiley and Sons, New York. 
Matsumoto K (1996) Establishment of Papilio demoleus L. (Papilionidae) in Java. Journal of Lepidoteran Society 50: 139140.

Meshram PB, Kulkarni N, Joshi KC (1996) Antifeedant activity of Azadirachta indica and Jatropha curcas against Papilio demoleus Linn. Journal of Environmental Biology 17: 295-298.

Murthy KSRK, Reddy AR, Kavuri Y (2007) Testing of Jatropha curcas seed oil on Spodoptera litura (Fab) for insecticidal properties. Pestology 31: 13-15.

Narayanamma VL, Savithri P, Rao R (2001) Influence of citrus butterfly, Papilio demoleus L. damage on growth parameters of the sweet orange host plant. Indian Journal of Plant Protection 29: $140-141$

Pandey R (2011) Bioefficacy of some medicinal plants and insecticides against Bombyx mori (Linn.) and Spodoptera litura (Fab.). Ph.D., Thesis submitted to the, G.B. Pant University of Agriculture and Technology, Pantnagar. 207 p.

Pandey S, Pandey JP, Tiwari RK (2012) Effect of Some Botanicals on Hemocytes and Molting of Papilio demoleus Larvae. Journal of Entomology 9: 23-31.

Sadek MM (2003) Antifeedant and toxic activity of Adhatoda vasica leaf extract against Spodoptera littoralis (Lepidoptera: Noctuidae). Journal of Applied Entomology 127: 396-404.
Sharma SS, Gill K, Malik MS, Malik OP (2001) Insecticidal, antifeedant and growth inhibitory activities of essential oils of some medicinal plants. Journal of Medicinal and Aromatic Plant Sciences 22: 373-377.

Singh SP (1993) Species composition and diapauses in citrus butterflies. Journal of Insect Science 6: 48-52.

Snedecor GW, Cochran WG (1967) Statistical methods. Oxford and IBH publishing Co., New Delhi. 593 p.

Srivastava KP (1993) A Text book of Applied Entomology, Vol 2, Kalyani Publishers, Ludhiana, India, Pp:120.

Tripathi AK (2002) Feeding deterrent and growth inhibitory effect of Lippia alba oil towards crop insect-pests. Journal of Medicinal and Aromatic Plant Sciences 24: 486-488.

Vaitheeswaran A (2009) Bioefficacy of some insecticides and insecticides mixtures against Spilarctia obliqua (Walker) and Spodoptera litura (Fabricius). M.Sc.(Ag.), Thesis submitted to the, G.B.Pant University of Agriculture and Technology., Pantnagar, pp: 89.

Vattikonda SR, Sangam SR (2016) Assessment of antifeedant potential of azadirachtin, a plant product of Azadirachta indica against Papilio demoleus L. (Lepidoptera: Papilionidae) larvae. Journal of Entomology and Zoology Studies: 956-959.

Yunus M, Munir M (1972) Host plant and host preference of lemon butterfly Papilio demoleus L. caterpillar. Pakistan Journal Zoology 4: 231-233. 